\title{
Reengineering Metaphysics: Modularity, Parthood, and Evolvability in Metabolic Engineering
}

\author{
Catherine Kendig* and Todd T. Eckdahl ${ }^{\dagger}$
}

\begin{abstract}
The premise of biological modularity is an ontological claim that appears to come out of practice. We understand that the biological world is modular because we can manipulate different parts of organisms in ways that would only work if there were discrete parts that were interchangeable. This is the foundation of the BioBrick assembly method widely used in synthetic biology. It is one of a number of methods that allows practitioners to construct and reconstruct biological pathways and devices using DNA libraries of standardized parts with known functions. In this paper, we investigate how the practice of synthetic biology reconfigures biological understanding of the key concepts of modularity and evolvability. We illustrate how this practice approach takes engineering knowledge and uses it to try to understand biological organization by showing how the construction of functional parts and processes can be used in synthetic experimental evolution. We introduce a new approach within synthetic biology that uses the premise of a parts-based ontology together with that of organismal self-organization to optimize orthogonal metabolic pathways in $E$. coli. We then use this and other examples to help characterize semisynthetic categories of modularity, parthood, and evolvability within the discipline.
\end{abstract}

\section{Keywords}

modularity $\bullet$ BioBrick assembly $\bullet$ evolvability $\bullet$ parts-based ontology $\bullet$ self-organization

Part of a special issue, Ontologies of Living Beings, guest-edited by A. M. Ferner and Thomas Pradeu

Editorial introduction: Catherine Kendig and Todd Eckdahl defend and illustrate a practice-based view of metaphysics of science. The target of their paper is the emerging and fascinating field of synthetic biology — a bioengineering domain that focuses on designing and assembling biological entities. The challenge they discuss is the following: What happens, ontologically-speaking, when as well as describing biological entities we start manufacturing new ones?

*Michigan State University, Department of Philosophy, Kedzie Hall \#503, 368 Farm Lane, East Lansing MI 48824, USA, kendig@msu.edu

${ }^{\dagger}$ Missouri Western State University, Department of Biology, 4525 Downs Drive, Saint Joseph, MO 64507, USA, eckdahl@missouriwestern.edu 
Kendig and Eckdahl examine the idea of modularity, which has long fascinated biologists, especially in evolution and 'evo-devo' (i.e., the articulation between evolutionary and developmental biology). They suggest that modularity is underpinned by an ontological claim about the living world. When we say that living things are 'modular,' we mean that they possess discrete parts that can be interchangeable. For the authors, modularity is an instance of a manipulation-based concept: the claim that a system is modular is in fact based on the observation that when we manipulate that system, it behaves as if its parts were discrete and interchangeable. Kendig and Eckdahl distinguish two concepts of modularity: 'global modularity' and 'local modularity,' and insist that if global modularity describes a system as modular, only local modularity can provide an explanation for the exact modularity the system displays. Examining two recent methods in synthetic biology (the BioBrick Assembly and the Golden Gate Assembly), they investigate how the practices of synthetic biology lead to a re-conceptualization of modularity and evolvability (defined as the capacity to change over time, by all sorts of natural, artificial, synthetic modifications).

This paper raises many critical metaphysical questions: What does it mean to 'exist,' and should we believe only in the existence of what we can manipulate? (This question, of course, was vividly raised by Ian Hacking-e.g., in his 1981 "Do we see through a microscope?" but is made even more pressing with the rise of synthetic biology). How do we define the 'parts' and the 'whole' in a system (see also Christopher Shields's contribution to this special issue)? Do engineered living beings belong to 'natural kinds'? In addition to 'in silico' systems (computer-based systems), can we use natural systems (such as bacteria) to solve some of our problems, particularly optimization problems? Perhaps most importantly, synthetic biology and bioengineering raise the following crucial questions: Is manipulability a criterion of reality - and if so in what sense?

$-\mathrm{AF} / \mathrm{TP}$

Synthetic biology research is based on an underlying ontological premise (i.e. that the world is structured in a certain way) that living organisms have an organization that is modular and that the variation that natural selection acts upon can result from the recombining of modules. The premise of biological modularity is an ontological claim that appears to come out of a practice. We understand that the biological world is modular because we can manipulate different parts of organisms in ways that would only work if there were discrete parts that were interchangeable. This is the foundation of the BioBrick assembly method widely used in synthetic biology (Knight 2003). It is one of a number of methods that allows practitioners to construct and reconstruct biological pathways and devices using DNA libraries of standardized parts with known functions (Brent 2004; Endy 2005). This construction and reconstruction, engineering and reengineering can be understood to be a general thesis of biological modularity based in practice. The parts database of biological modules with well-characterized functions relies on this working hypothesis, and the success of synthetically produced networks and parts in practice seems to bear this out at least in some limited capacity. A common application of synthetic biology is to manipulate the genetic control of metabolic pathways that either produce a useful metabolite, such as a biofuel or a pharmaceutical or destroy an unwanted one, such as an environmental toxin. Metabolic pathways that occur naturally in the host cell are referred to as 'native,' while introduced pathways are called 'orthogonal.' Native pathways can be used to provide starting materials for orthogonal pathways. The aim for engineering host cell metabolism is to integrate native and orthogonal metabolism.

In what follows, we investigate how the practice ${ }^{1}$ of synthetic biology may extend and shape

\footnotetext{
${ }^{1}$ For extended discussions of philosophy of science in practice see Hacking 1992, 1995; Dupré 1993;
} 
current discussion at the intersection of science and metaphysics by reconfiguring biological understanding of the key concepts of modularity and evolvability.

\section{Kinds of modularity}

As a multidisciplinary field, synthetic biology draws from genetics, biochemistry, organic chemistry, molecular biology, cellular biology, and microbiology. Because of its mixed disciplinary approach, it may be particularly well-suited to unpick the tangled meanings of concepts, such as modularity, that have been long-debated within evolutionary and functional biology (see Morange 2009a, 374). The concepts of modularity, constraint, and convergence can be stripped down and analysed and given functional parts-language descriptions through synthetic biological experimentation and manipulation of genomes of model organisms, such as that of the synthetic biology workhorse, E. coli. In addition to these concepts Morange mentions at play within synthetic biology in general, we suggest that the assembly methods used in metabolic engineering in particular can be used as an epistemic tool to also disentangle the multiple notions of evolvability and fitness.

The property of modularity has been widely discussed. Despite its seeming ubiquity within biological systems, what is inferred by the claim that an organism or biological system is modular needs some unpacking in order to be used in practice (see especially Schlosser and Wagner 2004; Wagner et al. 2007). Although understood to be a central principle of functional biology and synthetic biology, it is often described as a concept with multiple meanings. If this is the case, can we simply admit that the best approach to modularity is one of pluralism? If pluralism works for species concepts and gene concepts, why not modularity concepts? Sure, but this is not the problem that we identify here. Instead, our aim is to show that there is a lack of recognition that claims about modularity can belong to the category of modularity and there are claims that belong to the individuating concepts of modularity. Conflating claims about the category of modularity and those of individual concepts of modularity used to explain how the system is modular causes confusion. Within the emerging discipline of synthetic biology, this apparent ambiguity means that more than context is usually required for the use of the term to confer knowledge about the system being described as modular from one practitioner to another. We focus on a particular case and suggest the term 'modularity' is used to identify two different kinds of claims in the metabolic engineering of parts and pathways within synthetic biology. In doing so, we discriminate between what might be best described as the general philosophical claims of the category of modularity as 'global modularity' and we use 'local modularity' to refer to the more restricted claims of modularity that are set out within the separate operational concepts.

i. Global modularity (GM): A general category of properties of biological organization that claims that parts, processes, and pathways are made up of relatively independent components, like building blocks. This claim may take the form of a metaphysical category that refers to the properties of organization of living things as modular; a claim that applies with univocality to all biological structures included within it. It can be a methodological heuristic; that the best way to proceed with research is to look for modularity. It can be an epistemic heuristic, that the world is knowable and we gain understanding by considering it is modularly organized. The category of GM has been referred to variously

Chang 2004; Rouse 2003; De Regt, Leonelli, and Eigner 2009; Soler 2012; Soler, Zwart, Lynch \& Israel-Jost 2014; and Kendig 2016.

${ }^{2} \mathrm{We}$ are grateful to an anonymous reviewer for pointing to this problem. 
in the synthetic biology literature in terms of definitions: "modules are ... discrete entities, whose relatively autonomous functions are separable from those of other modules, and designed so that interconnecting modules allow higher level functions to be built" (Hartwell et al. 1999), and in terms of a concept: "[S]ynthetic biology begins with the concept of modularity. A system can be described as modular if its components can be functionally separated and recombined ... this abstractability makes it possible to do reductionist experiments in biology, isolating specific modules for close study to eliminate variables introduced by the cellular context" (Agapakis and Silver 2009, 704-705).

ii. Local modularity (LM): A specific claim about the parthood of a particular organism, pathway, device, or process. Local modularity is a restricted claim that refers to the domain specific properties of the object of concern and how they are integrated. It focuses on the propert(ies) of being a part, the individuation of parts, and the composition of parts as they relate to one another and to a particular whole. Included under this kind of claim are various sub-characterizations, for instance: that parts are relatively autonomous, interchangeable, independent of the context of other parts, carry out measurable functions, standardizable, or are non-decomposable. These are also included in GM in terms of descriptions of the general structure, but in LM techniques are provided to operationalize the measurement and manipulation of the interchangeability, independence and autonomy of modules. LM has been conceived of in a variety of ways depending on the system specified and the practitioners' interests to answer the question of how a system is modular: "the study of how genetic components work together to integrate environmental signals and effect a cellular response ... determine[s] what topological arrangement of regulatory modules can lead to a given behavior, often with strong analogy to electronic signal processing units such as logic gates and feedback loops" (Agapakis and Silver 2009, $705)$, or in terms of how biological entities interact: "biological parts and processes ... are organized in a modular fashion where regulatory molecules form groups with many interactions within a group and few interactions between groups" (Espinosa-Soto and Wagner 2010).

These two forms frame different spaces of epistemological and ontological investigation. In doing so, they configure the level of biological organization to which the theses of modularity (GM or LM) apply, and to what they apply.

Within synthetic biology, GM is used to conceptually frame the system one is investigating. GM is based on conceptualizing the system as modular whilst LM provides practitioners techniques by which to pursue their research goals (e.g., to make the system more modular and therefore more useable). Membership of a system within the category GM provides the practitioner a set of inferences based on heuristics of abstraction and standardization that inform her way of looking at a system. For instance, knowing the system is GM allows her to focus on parts with known functions that are stably expressed with minimal context sensitivity and irrespective of the other parts connected to them or in certain cases irrespective of the chassis organism used (see Andrianantoandro, Basu, Karig, and Weiss 2006). LM informs which engineering practices should be used by licensing the kinds of inferences the practitioner can make about the system under manipulation (Endy 2005).

\subsection{Assembly methods affecting modularity}

Synthetic biology is based on a general thesis of biological modularity (GM) (Knight 2003; Morange 2009a). The parts database of biological modules with well-characterized functions 
relies on this working hypothesis and the success of synthetically produced networks and parts in practice seems to bear this out at least in some limited capacity. In this section, we investigate how the practice of synthetic biology reconfigures biological understanding of GM and LM. We investigate how the assembly method one uses may affect the general claims about the nature of modularity of the parts, devices, and pathways.

The most commonly-used assembly method, BioBrick assembly, is based on the standardization of basic parts with a prefix and a suffix (Knight 2003). Addition of these two short DNA sequences to any DNA element results in a BioBrick. Two BioBricks can be ligated together in either order to construct a composite that is itself a BioBrick containing a prefix and a suffix. The central concept that enables BioBrick assembly to work is that type II restriction enzymes XbaI and SpeI produce compatible sticky ends. BioBrick assembly has been a popular method from its inception. However, it is not without problems. BioBrick assembly depends on purification of DNA fragments after gel electrophoresis, a time consuming method that is sometimes challenging even for experienced research groups. The pairing of the DNA recognition sequences for $\mathrm{XbaI}$ and SpeI during ligation results in a mixed site that practitioners refer to as a BioBrick scar. The scar is six DNA nucleotides in length and has a defined sequence. There are applications in which the spacing between two parts must be smaller than six nucleotides or when the BioBrick scar sequence confounds the function of a device. Another problem with BioBrick assembly is that once ligated together, two BioBricks cannot be taken apart for use in another construct. Assembled BioBricks are components that can no longer be swappable or interchangeable. In this way, the generation of the scar during BioBrick assembly affects the kinds of parts produced and their degree of modularity.

A newer method, Golden Gate Assembly (GGA) addresses several of the limitations of BioBrick assembly (Weber et al. 2011). GGA is based on the function of type IIs enzymes, such as BsaI, which bind to an asymmetrical DNA sequence and cut the DNA outside of the binding site. Two DNA parts to be connected by GGA are engineered by the practitioner to have BsaI restriction sites that produce complementary sticky ends. The DNA fragment on the left has a BsaI site directed toward a sticky end while the DNA fragment on the right has a BsaI site directed toward a complementary sticky end. The two DNA parts are mixed with both BsaI and DNA ligase simultaneously under conditions that support both enzymes. Ligation of the two DNA subparts results in an end product that contains no BsaI sites. GGA is more automated than BioBrick assembly since digestion and ligation are combined into a single reaction, avoiding purification of DNA fragments after gel electrophoresis. Another advantage is that the nucleotide spacing between two parts connected by GGA can be zero nucleotides rather than six nucleotides as in BioBrick assembly. Using GGA, parts have increased modularity when compared to those produced by the BioBrick method. In practice, 'modular' means something different for those using GGA than it does for those using BioBrick assembly.

GGA also has limitations. Because customized DNA primers must be designed and synthesized for each part to be assembled during GGA, the level of standardization of GGA is lower than it is for BioBrick assembly. GGA as originally described enables researchers to introduce genetic variation into a construct using small or large libraries of parts that vary in sequence and function, but not the reintroduction of variation into assembled constructs. This is a problem for any practitioner interested in the reintroduction of variation into populations of DNA constructs that have undergone functional selection. This approach is desirable because it more closely resembles evolution by natural selection than does the one time introduction of variation enabled by GGA. If the intention of the research is to make systems, devices, and pathways in synthetic biology that can be used to understand natural selection, then a new method is 
needed. Eckdahl et al. (2015) recently developed an adaptation of GGA called Junction GGA (J-GGA) that standardizes assembly and enables the high throughput introduction and reintroduction of genetic variation into DNA constructs. J-GGA employs a scaffold with junction sequences on each side of the parts within a gene expression cassette. The junctions enable the use of standardized primers for use in assembly. Variation can be introduced into combinations of genetic elements at the same time. J-GGA allows practitioners to introduce simultaneous variation of a number of different genetic variables, including transcriptional promoters, origins of replication that control plasmid copy number, ribosome binding sites, gene alleles, and protein degradation tags. Using J-GGA assembly, modularity both increases and is conceived of differently in practice than it is using either the BioBrick or GGA methods.

\subsection{Modularity and modularizing}

Whilst GM refers to the category of modularity, LM refers to different theses of discrete parthood (either of which may pertain to processes, products, relationships, or properties), and in particular, how biological systems, processes or pathways instantiate modularity.

When considering the different assembly methods BioBrick and GGA as described above, we find a problem if 'modularity' is taken to only refer to GM. The problem arises because it appears that knowledge of how a system is to be modular depends upon what techniques one uses, for example, what assembly method one uses. The category of modularity and the phenomenological state of being a module seems only to give us knowledge that the system is modular but not how it is. To understand how it is modular depends upon bioengineering practice. Being a module appears to not be defined in the GM category, e.g., in virtue of being a member of the category of being modular, or membership in some class, or based on the assumption that there are other properties that can be inferred from membership within the category. This is a problem if GM is conflated with LM insofar as GM defines being modular as something that is irrespective of the way in which modularity can be instantiated in a system or of assembly or tools used to make modules, whereas LM provides a way of understanding how modularity is instantiated.

The claim of LM is a claim specific to the compositional structure that parts and wholes exemplify in a particular system. LM is an answer to questions like: What is the nature of parts in that particular device or pathway? How are they organized? What is the relationship of the compositional structure of them to the whole in question? It characterizes the property and nature of modulehood in situ and so provides operational criteria that can be used to measure modularity within a system. The things that are categorized as modular are numerous and may come about due to differing processes of assembly or categorization. LM defines modules qua modules in their individuation and identification as parts of the pathway, process, or mechanism and in the satisfaction of a particular function or purpose. The assembly method used makes parts into modules insofar as they are chosen by practitioners to be connected or separated from other parts. What counts as a module (variously understood) and the criteria for modular kindhood may not be the same for all modules. That is, for LM, modularity may not be a property that can be univocally expressed for all parts. 3

However, LM is not without problems. To say something is a module or part has typically meant that it bears some sameness relationship, family resemblance, or overlapping shared homeostatic set of properties ${ }^{\mathbb{4}}$ to another part qua module. In this way, being a module-insofar

\footnotetext{
${ }^{3}$ In contrast, it is this universal claim that characterizes GM.

${ }^{4}$ Insofar as a module or part is something whose propert(ies) or relationship(s) are shared by other
} 
as it is understood to be a property - means that it is a property that is instantiable in one way. This means that all modules insofar as they are modules are homogeneously so. Local modulehood may be a kind differently instantiated. That is, modulehood for one thing may not be the same as modulehood for another in a radical and non-comparative way. This may be perceived a weakness of LM. Some might argue that this brings into question the legitimacy of what it is that we refer to when we claim something is a module if there is no unifying claim (like there is with GM). When faced with this proposition, some may prefer to dispense with all claims of modularity and become eliminativists. Alternatively, they may embrace the heterogeneity of local modulehood and allow that local modulehood may be a radically heterogeneous group of concepts that may overlap across all kinds of metaphysically parcelled out stuff and be LM pluralists while remaining GM monists. If GM and LM refer to different types of claimsthe former concerning the membership within a category and the latter a set of operational concepts for investigation of part-whole relationships that inform our understanding about the constituents of the universe- then they may both hold. One may retain the unifying category of GM whilst acknowledging that in LM, different assembly methods affect the degree of modularity and fungibility of parts such that what it is to be modular at the local level is variously understood.

\subsection{Description and explanation}

To say of a particular system that it is a member of the category GM is to say something about an abstract level of organization - that it is organized in some way so that it does whatever it does stably (Schlosser and Wagner 2004, 519-522; Wuensche 2004, 307-312). That is, GM licenses us to describe the system as having the property of modularity. LM provides a context specific concept that provides information about which resources are needed to make a system modular and how it is (or could be) modular or more modular. LM (unlike GM) provides a determination of modulehood that involves pragmatic and normative constraints that can be used to judge whether that thing (system process part) is modular or is not modular. Why is this important? How the property of being a module or the determination that a system has the nature of modulehood at a given time allows us to do things with it. If it is a module, and we know in what way it is modular, we may potentially be able to build things with it. Its being a module stands in a relation to us as practitioners in being a standardized part with a known function given a particular set of conditions. Describing something as a module makes something a resource for synthetic biology but it also provides us with a way of evaluating it as a particularly useful or particularly un-useful resource, e.g., if the function attributed to the standardized part is expressed reliably in a number of different systems-one that works in all chassis, or one that works in E. coli as well as S. cerevisiae - it might be thought of as particularly good.

Metabolic engineering relies on a part-whole relationship that is characterized in terms of the design and construction of standardized biological parts into synthetic biological wholes (Endy 2005). The standardization of parts is necessary to conceive of complex biological systems in ways that make these parts useable as tools for synthetic biology practitioners. But doing so also relies on knowing the appropriate engineering practices necessary for the construction of processes, devices, and mechanisms which in turn relies on understanding how a system is modular. That there are modules is descriptive of a system, knowing how things are modular

things referred to as the same kind of part, the nature of parthood can be understood in terms of kindhood. See Putnam (1975), in particular his use of the conception of a sameness relation, Wittgenstein's (1953) use of family resemblances, or Boyd's $(1991,1999)$ homeostatic property cluster kinds. 
provides an explanation of its modularity. The latter requires investigation into the integration within modules themselves as well as the integration (or nonintegration) between modules in such a way that the system works (cf. Nandagopal and Elowitz 2011, 1244-1248). It makes the concept of modularity useable by providing ways of measuring or tracking it in systems. For instance, Del Vecchio et al. (2008) conceptualize modularity by relying on the notion of retroactivity ${ }^{[}$which they expand and operationalize in their clarification of the criteria for modularity. For Del Vecchio et al., a system is made up of nodes where each node is considered retroactive in so far as there is an effect on the upstream process that is caused (or effected) by a downstream process. Retroactivity can be measured for every node in the system or pathway (e.g., a metabolite). In this way, nonmodularity and modularity are defined in terms of retroactivity and the absence of retroactivity, respectively. A module can be identified as that node that does not have reactivity and a node that does have reactivity is one that is not a module (Del Vecchio et al. 2008).

Whereas GM offers a description of a system as modular, LM provides an explanation. How a system instantiates the property of modularity means investigating how the different parts cohere as modules and how they interact with other modules. This is dependent on how these are individuated in respect to the normative, epistemic, and methodological constraints that frame the role of the part as a module and frame it as a resource for synthetic biology practitioners within a particular local concept of modularity. The cause of the modularity must always be provided by the local conception that is specified in the context of the object to be investigated and the investigator.

We might say that GM is of value as a heuristic to investigate the possibility of module-like things. It might be conceived of as a necessary premise prior to further investigation - that something may be a module or as a putative property of a system which may or may not turn out to actually be modular. That is, it may frame research as being towards the goal of finding or identifying a module. But if GM is of this kind of heuristic value, it might be because we infer that if the system is modular, then it must be modular in a particular way. If one is thinking in terms of a general approach that sees systems as modular, one may say what one is doing is just thinking about things modularly. Within metabolic engineering, thinking about modules doesn't get us where we need to go. What we really want to do is to find them locally and know how they work. LM is what allows the practitioner to uncover the techniques and resources by which she can reengineer a system with success (knowing that the function of the module will work in the chassis she chooses because it has worked in others reliably). But this knowledge itself arises from manipulating the modules within the systems in a way that is productive or towards that goal. It comes from practice. For instance, if what is sought is a way to make the pathway stable, one might restrict the focus to those parts which the practitioner knows were manipulable in the system in the last experiment. Doing this means that the kind of explanation sought directs and is directed by the investigator.

Seeking a cause or a number of causes for why some system has the property of modularity means deciding to focus on a particular set of causes that are believed to be informative for a particular explanatory purpose whilst others are ignored (cf. Shaffer 2005). This is an old problem-how do we decide what to include in our explanation from our knowledge in order to answer the question we are asking in a way that is explanatorily salient? (see Kitcher 1981; van Fraassen 1980; Waters 2007; Oftedal and Parkkinen 2013). Oftedal and Parkkinen (2007) suggest that this problem is even worse in synthetic biology because rather than just looking for causal explanations of biological systems that exist and are there to be investigated, practitioners

\footnotetext{
${ }^{5}$ Retroactivity is a concept introduced by Saez-Rodriguez et al. 2005 .
} 
are constructing the phenomena (and the explanandum) that they are seeking to explain. That is, practitioners "manipulate[e] biological systems, building on the explanatory resources available for understanding them" (Oftedal and Parkkinen 2013, 212). Practitioners frame an explanandum within a system of practice where parts are constructed in a controlled environment and where causal stability is both maintained and sought - doing so directs the kind of engineering practices and methods used to reengineer the metabolic pathway and may lead to understanding of the system that provides explanatory resources for future manipulation. We conclude with the suggestion that the concepts of modularity used within LM shape the questions being asked about the modularity of systems as well as the resources used to answer them. LM defines parts, the relationship of parts to other parts within the modular system and the relationship of modules to other modules (e.g., their interreactivity within a system of modules). What a module is, is in some way always a 'module-for' in two senses-it is a module that functions in a particular way given the system of investigation, and it is a module that is defined in terms of the investigator who uses it and her interests and goals in its use.

\section{How Can Modularity Help Facilitate Evolvability?}

We move now from mapping out some of the conceptual terrain of modularity within synthetic biology to that of evolvability. What is evolvability? We think of natural organisms as evolvable in terms of their ability to adapt and one of the factors that facilitates this adaptability is thought to be the ubiquity of modular networks. As such, many define what it is to be a module in terms of its evolvability, as a "semi-autonomous entity that evolves, functions or participates in development (or other processes) relatively independently from other modules" (EspinosaSoto and Wagner 2010, 1). But how or why modularity evolved remains an open question (see especially Clune et al. 2013). Some argue that modularity is a property that emerges as a side effect, or byproduct, of evolution: "modularity can increase in gene regulatory networks as a byproduct of specialization in gene activity" (Espinosa-Soto and Wagner 2010, 1). Whilst others argue that it drives phenotypic variation: "Modular organization is a universal property of phenotypes, the result of the universally branching nature of development. Switches divide the phenotype into somewhat discrete and semidissociable subunits of structure and gene expression or gene product use at all levels of organization" (West-Eberhard 2003, 56). One of the most influential accounts of the role modularity plays in evolution focuses on this notion of dissociability. Dissociability is a property that facilitates variation through compartmentation where compartmentation is understood to be some degree of individualization (Kirschner and Gerhart 1998, 2005). On this view, modularity is central to facilitated variation by allowing systems the capacity to maintain modular function in one area whilst making changes that do not have upstream effects. As a property of a system that affords evolvability it has also been more recently described as the property of near-decomposability (Wimsatt 2009) and semi-autonomy (Espinosa-Soto and Wagner 2010). But how exactly can modularity help facilitate evolvability? Weak linkage between modules allows one feature of the system to dissociate from it and may then be used in association with a different trait. This 'reconfigurability' of modular features (as subunits of traits) constitutes a highly conserved core capacity of organisms (Kirschner and Gerhart 2005, 136). As such, it furnishes the possibility of dissociating these modular units and their subsequent rearrangement into novel combinations by the biological system to serve other purposes. The compartmentalization of the component parts of an organism into relatively discrete units or subunits means that the effects of variations in one subunit may be isolated from those of another (see Kirschner and Gerhart 2005). If these are, in turn, inherited as subunits, 
the modularity of the organism may increase over generations.

\subsection{Engineering evolvability}

How can evolvability be characterized in synthetic biology? We suggest that it may be best understood as the capacity of a population, organism, device, part, or pathway to change over time-that is, evolvability is the retained space of variability of self-organized systems. Facilitated variation can then be understood to be the organization and reorganization of modular parts necessary for evolvability more generally. On this view, modularity is a property of both engineered and native systems for the same reason. If a biological system is modular, it is able to make changes in downstream nodes without effects upstream, so change can occur in one area without affecting the whole metabolic pathway and the system (or the practitioner) can utilize resources in new combinatorial strategies. Conceived of in this way, it can serve as an umbrella term under which natural, artificial, and synthetic change over time can be covered.

In the remaining sections, we discuss the problem of natural selection for metabolic engineering and a possible solution. The solution relies on a particular example of metabolic engineering, its potential for harnessing evolvability in synthetic biology, and conceptual consideration of it as a new mechanism of evolvability pertinent to the practice of synthetic biology. We consider whether evolvability can be engineered and how we might categorize these (and other) kinds of evolutionary change within synthetic biology. In particular, we suggest that investigating the methods and practices of knowledge acquisition within synthetic biology presents an alternative way of exploring metaphysical questions within philosophy such as: What are the kinds of things and processes that exist and the scientific categories to which they belong? What is the relationship between parts and wholes? It also introduces new questions about what it is to be a part or indeed the property of partness itself; questions whose answers seem to rely on understanding the metaphysics of science as grounded in the actual practice of it. We suggest a categorization of the products of some of these methods as semisynthetic to mark these out as new routes of the evolution of novelty and innovation that are the result of combined native and orthogonal pathways.

\subsection{Current research and applications}

To explain the potential for harnessing evolvability within metabolic engineering, we need to refer actual examples of this and outline the kinds of processes involved in the reengineering of metabolic pathways. We begin by briefly describing a series of recent studies focusing on the metabolic engineering of yeast cells for production of plant-derived metabolites (DeLoache et al. 2015) before discussing the problems of optimization and natural selection that arise for this and other studies (see Table 1) focused on metabolic engineering. DeLoache et al. sought a way to engineer yeast cells to produce pharmaceuticals in a chemical family called benzylisoquinoline alkaloids (BIAs). BIAs include the analgesics morphine and codeine, the antibiotics sanguinarine and berberine, the muscle relaxants tubocurarine and papaverine and the cough suppressant

\footnotetext{
${ }^{6}$ For a recent discussion of evolvability and synthetic engineering that is complementary to the one presented here, see Calcott 2014).

${ }^{7} \mathrm{We}$ introduce the neologism of 'semisynthetic evolution.' We believe that this is a justified and appropriate descriptor as the evolution described arises in part due to the manipulation by synthetic biologists and in part through (non-human) selective forces. It is hoped that this classification will highlight the potential role for discussion of different kinds of evolution due to the different causal mechanisms by which they occur
} 
Table 1: Modes of development and aspects of organization to which stem cells contribute.

\begin{tabular}{llll}
\hline Host Organism & Metabolite & Application & Reference \\
\hline Yeast & Artemisinin & Anti-malarial drug & Martin et al. 2003 \\
E. coli & organic acids & Chemical commodities & Yu et al. 2011 \\
E. coli & Isobutyl alcohol & Biofuels & Atsumi et al. 2008 \\
Bacterial consortium & Copper & Bioremediation & Brune, Bayer 2012 \\
\hline
\end{tabular}

noscapine. Extensive research on the production of BIAs in plants such as the opium poppy yielded information about the native metabolism, genes involved, enzymes they encode, and the metabolic pathways they control. Efforts to engineer $E$. coli for BIA synthesis led to the production of the intermediate metabolite $(\mathrm{S})$-reticuline in a combined native and orthogonal pathway but failed to produce BIAs in downstream metabolic steps. The engineering of yeast cells able to carry out these downstream steps indicated that eukaryotic yeast cells may be better equipped for BIA metabolic engineering than prokaryotic $E$. coli cells. Understanding the native metabolism, this makes sense considering that naturally occurring BIA synthesis occurs in eukaryotic plants.

Although some BIAs have been produced by coculturing E. coli for (S)-reticuline production with yeast for BIA synthesis, this strategy does not scale well for industrial pharmaceutical settings. In response, DeLoache et al. sought to engineer the production in yeast of $(\mathrm{S})$-reticuline using orthogonal downstream metabolic steps to produce BIAs. The group directed the protein evolution of a key enzyme in the pathway to $(\mathrm{S})$-reticuline by introducing mutations into the gene coding for the enzyme and screened for a mutant enzyme that functioned with high efficiency. They were able to use the mutant enzyme to complete a seven step pathway in yeast from the simple sugar glucose to $(\mathrm{S})$-reticuline. Their result affords the opportunity to combine the metabolic steps leading to $(\mathrm{S})$-reticuline with those already demonstrated to convert (S)-reticuline to BIAs. Doing so would suggest both the feasibility of using microbes and the possible utility of metabolic engineering of yeast cells to produce valuable BIAs.

Table 1 shows four other examples of microbial metabolic engineering. The most high profile example resulted in yeast cells that produce the anti-malarial drug artemisinin, reducing its cost of production compared to purification from the sweet wormwood plant (Martin 2003). Metabolic engineering of $E$. coli enabled the production of succinic acid, lactic acid, and glucaric acid as chemical commodities (Yu et al. 2011) and isobutyl alcohol as a biofuel. A bioremediation application involved the recovery of copper using a consortium of metabolically engineered bacteria.

\subsection{Two problems: optimization and natural selection}

Despite the successful reengineering of yeast cells to produce (S)-reticuline (DeLoache et al. 2015) and in the production of the anti-malarial drug artemisinin (Martin et al. 2003); E. coli enabled to produce of succinic acid, lactic acid, and glucaric acid as chemical commodities ( $\mathrm{Yu}$ 2011); and consortia of bacteria for bioremediation (Brune and Bayer 2012; see Table 1), two problems keep arising. We will refer to the first of these as the optimization problem: How can synthetic biologists optimize orthogonal metabolic output when they cannot know all of the variables that affect it in a host organism and its environment? We call the second problem the natural selection problem: How can the reduction in fitness in a population of organisms engineered for an orthogonal metabolic pathway be prevented from causing evolution away 
from the desired metabolic output phenotype?

The native metabolism of all organisms is responsive to a vast array of internal and external variables including genotype, metabolite availability, $\mathrm{pH}$, osmotic pressure, and temperature. When synthetic biologists introduce orthogonal metabolism into a host organism, they can optimize metabolic output by taking into account the variables they know, but must ignore all of the variables they don't know and some that they cannot know. Practitioners also struggle with the difficulty of trying to make sure that the organisms they engineer with the ability to carry out orthogonal metabolism continue to faithfully replicate that genetic capacity. If the production of the product or the maintenance of the pathway is too onerous for the cell, the metabolic pathway will (after a number of generations) be disposed of. Those organisms without the onerously produced product or taxing pathway will be selected for in preference to those constructed to function by the synthetic biologist.

To address the optimization problem, practitioners have typically relied on what is called a "rational approach" (Boyle and Silver 2012). This approach aims to optimize a metabolic pathway by using knowledge of cellular (e.g., bacterial) physiology. Insofar as our understanding of bacterial physiology and the effects of synthetically constructed pathways within $E$. coli or another biological chassis is limited, so too are the efforts to constrain the pathway in light of unknown (or perhaps unknowable) variables that may affect the output of a particular engineered metabolic pathway. This problem-solving approach of the introduction of orthogonal metabolism, measurement of metabolic output, and tweaking of pathways to improve output has been the standard approach to the optimization problem.

An alternative to the rational approach is the peculiarly named "rationally irrational approach" (Boyle and Silver 2012). Whereas the rational approach meant attempting to use know variables to reason an optimal combination of genetic elements controlling metabolism, the rationally irrational approach means using selection to solve the optimization problem. The rationally irrational approach takes into account the known variables as well as the unknown variables at play in the regulation of orthogonal metabolic pathways. These variables include extracellular and intracellular $\mathrm{pH}$, osmolarity of the media, stress caused by orthogonal metabolism and endogenous metabolic flux. The effects that they have on orthogonal metabolism are difficult to control.

Recently, Eckdahl et al. (2015) employed the rationally irrational approach in what they refer to as 'programmed evolution,' utilizing a different kind of computation to traditional siliconbased computation. Their strategy takes the heritable space of variability of self-organized systems and uses it to try to resolve the as-yet intractable problem of natural selection described at the beginning of this section. Their programmed evolution abstracts bacterial cells as living computers capable of taking many chemical and physical inputs and processing them with gene expression algorithms to regulate their metabolism. It uses bacterial computers to compute solutions to a metabolic optimization problem chosen by the practitioners. Cells are programmed with variation in genetic control elements that control the gene expression required for the desired metabolic pathway. The bacteria acquire inputs that are unknown to the researchers. The bacteria will process all of this information as living analog computers, using the results to direct the operation of their biochemical hardware. Eckdahl et al. justify their motivation for using bacteria to do this computing by suggesting that bacteria are better informed and more capable of making these calculations than people and the silicon computers they program with incomplete information and models. Optimization of the metabolic pathway is achieved by the

${ }^{8}$ Although distinct, A. Wagner's 2011 approach to evolutionary innovation as an exploration of a space of possibilities is similar to that used by Eckdahl et al. 2015. 
bacterial population through natural selection. Once genetic variation is introduced into the bacterial population, selection is imposed on it. Fitness is defined by the ability to produce the most of the desired metabolic product. After selection, the allele frequency in the population will have changed, effecting evolution of the bacterial population. Successive cycles of genetic variation and selection cause the population to continue to evolve. Repeated generations of evolution are expected to lead to increased optimization of the metabolic pathway. Coupling fitness to optimal metabolic output also prevents the population from evolving away from optimal product formation.

Before addressing the hard question of how evolvability and modularity are connected, we make the provisional suggestion that one way evolvability and modularity can be connected is in terms of this coupling of fitness to optimize metabolic output. Reengineering the retained space of possible variability for this purpose - as the work of DeLoache et al. (2015) and Eckdahl et al. (2015) shows-harnesses the power of self-organizing systems. In doing so these approaches to metabolic engineering direct or program evolution by using natural selection itself to solve the selection problem by co-opting the problem-solving and computing abilities inherent in the bacterial population that is the object of study. That is, it enables researchers to program bacteria and use evolution to compute the optimal combination of genetic control elements that maximizes metabolic output and to maintain it in a population of cells.

The aim of synthetic biology in this mode is to allow the bacteria themselves to identify properties of the system that increase their evolvability by facilitating their own variation given certain inputs from practitioners. Bacteria are the ones able to combine resources in the best way to optimize the metabolic pathway. Rather than use silicon-based computers and software to compute the best functionality and evolvability of the biological system, programmed evolution uses bacterial computers to solve the optimization problems.

This research shows that the introduction of genetic variation into a synthetic biology chassis organism and imposition of user-defined selection to optimize an orthogonal metabolic pathway are possible. In doing so, it seems to suggest a generalizable method. This new method allows researchers to input a large number of variables rather than single variations. In doing so, it replicates something that approximates native evolution. The result is a unified native-orthogonal method that aims to solve - rather than offering a disjointed number of orthogonal solutionsthe optimization of metabolic pathways. It does so by allowing bacterial cells to do the heavy lifting (and intentional construction) for themselves by taking into account the variables that are unknown to the researcher. E. coli are allowed to solve the problem of using a variety of chemical and physical inputs together with a gene expression algorithm to regulate their biochemical hardware. The rationally irrational approach is used to employ natural selection in the optimization of orthogonal metabolic pathways by coupling metabolic output to cellular fitness. Bacterial cells use chemical and physical inputs (provided by the practitioners) and process them in a way that is optimal given certain fitness constraints. For instance, Eckdahl et al. (2015) employed $E$. coli to optimize a metabolic pathway for the conversion of caffeine to theophylline. Twenty-four strains were constructed that carried combinations of multiple genotypic variables. These included the strength of the promoter, the ribosomal binding site, plasmid copy number, and chaperone proteins. Fitness was linked to theophylline production by using a theophylline riboswitch and a tetracycline resistance protein. This allowed researchers to measure change in the distribution of the various twenty-four genotypes within the E. coli population (Eckdahl et al. 2015). 


\section{What Is the Connection Between Evolvability and Modularity?}

In the foregoing sections, we suggested that the methods that are used within synthetic biology affect certain properties of biological systems, namely modularity and evolvability. But we have not addressed a hard question-what is the connection between modularity and evolvability? Evolvability of systems may (in some but not in all cases) be affected by the kind of modularity that is exhibited or constructed within the system. Our remaining discussion focuses on how the properties of an organism affect the changes in its metabolic pathway over time. It provides a unique set of justifications for exploring engineering practices within synthetic biology that may reveal the nature of modularity and evolvability. It is hoped that these will complement the work of Karthik Raman and Andreas Wagner (2011) on the programmability of digital circuits and their evolvability over time.?

If modularity can be conceived of in terms of either GM or LM (or both) in metabolic engineering, what is the connection between evolvability and modularity? We suggest that the answer to this question relies on the identification of which parts or pathways have conserved tinkerability. 'Tinkerability' can be understood as the property of biological parts that makes them better suited to repeated modifications over generations. Biological parts acquire the property of tinkerability either through the process of evolution (following Jacob 1977), or through the repeated manipulation by engineers. If tinkerability is a property that is conserved over generations, it is a prerequisite of evolvability. That is, its retention is itself a property of evolvability (cf. Foote and Yoder 2000, discussion on software design). Evolvability is an evolved property, but how it evolves modularity is a question pursued both within synthetic biology and outside of it (Clune et al. 2013). Modularity is one structure (but not the only one) that evolvability is manifest in biological systems. So the answer to the question: what is the connection between evolvability and modularity? Is: modularity is a property that affects how metabolism can change over time and throughout a lineage. It provides (either by the organism or the engineer, or in the cases we have discussed, both) multiple ways of optimizing the metabolic pathway.

\section{Is semisynthetic evolvability still evolvability?}

We return now to discuss evolvability. Earlier we suggested that evolvability may be understood as the capacity of an organism, device, part, or pathway to change over time-that evolvability is the heritable retained space of variability of self-organized systems. Conceived of in this way, evolvability was able to be used as an umbrella concept under which natural, artificial, and synthetic change over time can be covered. We now want to question the legitimacy of this as a category or kind in practice.

Synthetic biological research, as well as the products and processes that it creates, sits in a liminal position between engineered technological kinds and natural biological kinds (see Schyfter 2012). This has been seen as a difficult or messy position to be in with regard to kindhood and discipline-building insofar as that to which a discipline focuses its research and questions on is delimited by the kinds that define it. The notion that we have outlined in the foregoing discussion of conserved spaces of variability and programmed evolution-that evolvability is something that can be engineered and modified-has typically been dismissed as a category

\footnotetext{
${ }^{9}$ See also Wagner 2011 for the use of engineering metaphors in the investigation of evolutionary systems biology and Calcott et al. 2015; Kashtan and Alon 2005; Foote and Yoder 2000 for more on evolvability and modularity.
} 
mistake:

evolvability [is] not just a feature that can be added to the list of other technical specifications, because [it] constitutes[s] an undefined dynamic challenge that precisely forbids the application of the methods of design and implementation that engineers have practised so far (Marlière 2009, 83).

We challenge this. The recent study of programmed evolution and other research using a "rationally irrational approach" (Boyle and Silver 2012) suggests that evolvability is something that not only can be engineered and modified, but it has been engineered and modified. Insofar as the engineering is partially done in-house by the bacteria themselves, this may constitute a Gestalt switch in engineering practice and design. Both the practice and product of programmed evolution can perhaps be accurately referred to as 'hybrid,' or as we've suggested, 'semisynthetic' category.

Looking back to the various kinds of synthetic biology research and their concomitant epistemological categories, the rationally irrational approach and the co-opting of bacterial computing to optimize metabolic pathways brings forward a new category of synthetic biology investigation. It is similar to protocell creation (O'Malley et al. 2008) in seeking to understand the process of evolution, biological organization, and the nature of modularity and evolvability. 10 But the goal of understanding this process is directed to the potential to modify and optimize the modules, networks and systems which direct it. It takes evolvability to be a capacity that can be defined, represented, measured, intervened upon and optimized by harnessing bacterial populations as biological computers. Semisynthetic evolvability is the change over time of rationally irrational engineered populations. It differs from native or orthogonal evolvability in that the synthetic biologist actively intervenes in the process of variation and employs the use of a suite of constructed parts and employs populations of organisms to solve the biological problem of optimization.

But are semisynthetic evolvability and the umbrella conception of evolvability legitimate and veridical categories of evolvability? And how are we to judge them to be so? According to Morange, "Selection in nature and evolution directed by biochemists and molecular biologists increasingly differ as technological progress is made in synthetic biology" (Morange 2009a, 370). At first blush, this would suggest that our semisynthetic evolvability may not count as evolution at all. Later on, however, Morange provides an alternative account. He argues against his initial suggestion that synthetically tweaked evolution does not count as evolution. He goes on to argue that current understanding of evolution is based on an unwarranted assumption of univocalism that could be challenged by findings in synthetic biology:

some of the extraordinary scenarios of evolution, designed by synthetic biologists, must be carefully considered by evolutionary biologists. Maybe the models provided by evolutionary biologists are too restrictive, leaving too much place to uniformitarianism, the hypothesis that the mechanisms of evolution have always remained the same (Morange 2009a, 374).

Put another way, perhaps evolutionary biologists have just been getting it wrong. Native evolvability is actually more like our semisynthetic evolvability. So research into the latter may yield understanding of the former. Judged according to Morange's second suggestion, our umbrella category of evolvability can also be viewed as veridical insofar as it captures the heterogeneity of

\footnotetext{
${ }^{10}$ See O'Malley et al. 2008 for a discussion of the categories of knowledge-making in synthetic biology, one of which they refer to as "protocell creation."
} 
synthetic, artificial, and natural evolutionary mechanisms as well as their concomitant epistemic categories.

\section{Modulehood as a Property of Agent-Centered Artifacts}

The objects of synthetic biological investigation and manipulation are living systems. It appears to be a discipline for which the functional devices, parts and pathways, the engineering practices, and the epistemological and ontological categories to which they belong are transforming. So, what can we say about the nature of these categories? What are synthetic biological kinds? and, What kinds of kinds are the kinds within the discipline of synthetic biology? Schyfter (2012) suggests what he calls an initial "exploration into things and kinds" offering a "first look analysis" of kindhood for the products of synthetic biology and suggests that they fit imperfectly within both technological kinds and natural biological kinds. 11 He is critical of synthetic biologists for not considering kindhood and for using engineering as the model and exemplar on which to base synthetic biology. We suggest that the imperfect fit of the products of synthetic biology is because that which is kinded is heterogeneous. If synthetic biology provides knowledge of how systems work, then the explanations of nature it provides (e.g., about how to re-engineer and manipulate them; cf. Sprinzak and Elowitz 2005; Keller 2009) suggest that it may be more profitably conceived of as a discipline that is epistemologically, ontologically, and methodologically hybrid. Semisynthetic kinds can be simply understood as hybrid organisms, parts, pathways, devices, or modules that are partially the result of natural selection and partially the result of engineered manipulation.

Instead of seeking to understand the use of epistemological and ontological categories in practice from the premise that their existence can be known a priori or contained within the theoretical framework of the discipline that uses them, this approach runs in the opposite direction. Categories come into being in practice and from these categories-in-use theoretical concepts, notions of causal directionality, functional architectures, and other relata arise (see Kendig 2016b for an extended discussion of how these categories arise in synthetic biology). A weak version of this approach that explicitly focuses on practice connects knowledge with its concomitant mode of research. Practice is ineliminable. It does more than shape knowledge, the practice of reengineering or making is knowing (Keller 2009, 377). A (problematically) strong view of this would hold that theoretical concepts do not underpin and are not prerequisite to empirical investigation. They follow rather than precede it. 12

\section{Conclusion}

In the foregoing, we aimed to extend philosophical discussion within synthetic biology by focusing on the study and investigative practices used within it and on how practices in metabolic engineering might suggest new approaches to the concepts of modularity and evolvability. Referring to a series of recent studies, we investigated the methods of assembly used and the evolu-

\footnotetext{
${ }^{11}$ Schyfter (2012) considers and evaluates the appropriateness of conceiving the products of synthetic biological research as kinds of technological objects. Our discussion differs from his insofar as we take a practice-based account of kinds that focuses on kinds of modules.

${ }^{12}$ Reference to Richard Feynman's "what I cannot create, I do not understand" may seem appropriate here. However, many have carefully pointed out that Feynman's views about knowledge were largely inconsistent with the typical reading of what is often referred to as the 'chalkboard quote' and so taking it as exemplar to his thinking is problematic (O’Malley 2009; Keller 2009).
} 
tionary mechanisms retooled to overcome natural selection against metabolically reengineered systems once made. We concluded by suggesting the potential need for new categories of modularity and evolvability. What has been left out of the present discussion is role of the practitioner. We suggest this as a potential area of further research. One way to carve up semisynthetic kinds as kinds that are partially engineered technological kinds and partially natural biological kinds is to take modulehood to be a property of agent-centered artifacts. Semisynthetic kinds, such as the engineered pathways and processes discussed in the above, is to consider them as objects that are used for practical purposes (see Houkes and Vermaas 2013; for criticisms see Longy 2013). Taking an agent-centered approach, the individuation of modules as modules would be understood as relying on shared agency of the community of practitioners, knowledge of affordances or capacities, and the well-defined functions of parts, pathways, processes, and mechanisms that are considered potential resources to be used in the design of a new device. In this way, local modularity rests - at least in part-on the values of the practitioners and their intentions. Some might suggest that this amounts to a rejection of semisynthetic kinds as natural or even as seminatural kinds. We suggest that the intentional categories of practitioners are natural insofar as they partially individuate the etiological function of the engineered device under construction. In doing so, the engineer normatively constrains the function of the device.

So what about GM? Some might argue that GM doesn't really do anything for us as it doesn't inform practice and so calling attention to the lack of recognition between the category of modularity and how it works might be met with the response, so what? So, what are GM and LM doing for us? GM provides us with a heuristic claim of modularity (that a system is modular). LM provides a means of addressing two separate but overlapping questions: the question of why modularity exists-e.g., the ubiquity of modularity as explanandum (why does it happen, what's it for?), and modularity as an explanans in how it leads to evolution (or as some suggest, how it emerges from evolution). The role of GM and LM in synthetic biology can be understood as a mode of thinking that allows us to conceive and measure a system as potentially decomposable and re-orderable relying on the decomposability perspective which works best for that system. In some sense this can be understood to be the implementation of different notions of what could be referred to as 'reductionisms-in-practice,' which refer to the different possible ways of partitioning wholes into parts (Wimsatt 2009, 159-312). Reductionisms-in-practice characterize the system in terms of different conditions modes of organizational independence. Each yield "an approximate and adjustable framework [that allows] one to construct orderings for how well each condition [of invariance] is met ... across different decompositions of a system into parts ... the better a decomposition meets these conditions, the more nearly it factors the system into modular parts that can be characterized from that perspective into terms of monadic, intrinsic, or context-independent properties" (Wimsatt 2009, 176). Our approach explains why these partitionings of the system work in terms of LM concepts and how these concepts are based in and are shaped by practice. It is through iterative experimental practices that synthetic biology practitioners reengineer both metabolic modular devices, but also the operational and normative constraints necessary to make sense of why and how the system is modular. These provide further adjustments and valuations of the usability of modules for their particular goaldirected purpose, inform future investigations, and explain the nature of modularity within that system. 


\section{Acknowledgments}

We gratefully acknowledge financial support for this research from the National Science Foundation, Division of Molecular and Cellular Biosciences (MCB), (RUI: BIOMAPS: Modular Programmed Evolution of Bacteria for Optimization of Metabolic Pathways, Grant No. MCB-1329350 and Research Opportunity Award, Amendment No. 001, Proposal No. MCB-1417799). C. Kendig also thanks the Center for Philosophy of Science, University of Pittsburgh, for a visiting research fellowship for 2017 which supported the writing of the paper, and the participants at the 2016 Philosophy of Biology at Madison (POBAM) workshop for critical comments on an earlier presentation of these ideas.

\section{Literature cited}

Agapakis, C., and P. Silver. 2009. "Synthetic Biology: Exploring and Exploiting Genetic Modularity Through the Design of Novel Biological Networks.” Molecular BioSystems 5: 704-713.

Andrianantoandro, E., S. Basu, D. K. Karig, and R. Weiss. 2006. "Synthetic Biology: New Engineering Rules for an Emerging Discipline." Molecular Systems Biology 2: 0028.

Atsumi S., T. Hanai, and J. C. Liao. 2008. "Non-Fermentative Pathways for Synthesis of BranchedChain Higher Alcohols as Biofuels.” Nature 451: 86-89.

Boyd, R. N. 1991. "Realism, Anti-Foundationalism and the Enthusiasm for Natural Kinds." Philosophical Studies 61: 127-148.

Boyd, R. 1999. "Homeostasis, Species, and Higher Taxa." In Species: New Interdisciplinary Essays, edited by R. A. Wilson, 141-185. Cambridge, MA: MIT Press.

Boyle, P., and P. Silver. 2012. "Parts Plus Pipes: Synthetic Biology Approaches to Metabolic Engineering." Metabolic Engineering 14: 223-232.

Brune, K., and T. Bayer. 2012. "Engineering Microbial Consortia to Enhance Biomining and Bioremediation.” Frontiers in Microbiology 3: 203. doi:10.3389/Fmicb.2012.00203.

Brent, R. 2004. “A Partnership Between Biology and Engineering.” Nature Biotechnology 22: 12111214.

Calcott, B. 2014. "Engineering and Evolvability." Biology and Philosophy 29 (3): 293-313.

Calcott, B., A. Levy, M. Siegal, O. Soyer, and A. Wagner. 2015. "Engineering and Biology: Counsel for a Continued Relationship." Biological Theory 10 (1): 50-59.

Chang, H. 2004. Inventing Temperature: Measurement and Scientific Progress. New York: Oxford University Press.

Clune, J., J.-B. Mouret, and H. Lipson. 2013. “The Evolutionary Origins of Modularity.” Proceedings of the Royal Society B: Biological Sciences 280 (1755): 20122863.

DeLoache, W., Z. Russ, L. Narcross, A. Gonzales, V. Martin, and J. Dueber. 2015. "An EnzymeCoupled Biosensor Enables (S)-Reticuline Production in Yeast From Glucose." Nature Chemical Biology 11: 465-471.

Del Vecchio, D., A. Ninfa, and E. Sontag. 2008. "Modular Cell Biology: Retroactivity and Insulation." Molecular Systems Biology 4: 161.

Dupré, J. 1993. The Disorder of Things: Metaphysical Foundations of the Disunity of Science. Cambridge, MA: Harvard University Press.

Eckdahl, T. T., A. M. Campbell, L. J. Heyer, J. L. Poet, D. N. Blauch, N. L. Snyder, et al. "2015 Programmed Evolution for Optimization of Orthogonal Metabolic Output in Bacteria.” PLoS ONE 10(2): e0118322. doi:10.1371/journal.pone.0118322. 
Endy, D. 2005. “Foundations for Engineering Biology.” Nature 438 (24): 449-453.

Espinosa-Soto, C., and A. Wagner. 2010. "Specialization Can Drive the Evolution of Modularity." PLoS Computational Biology 6:e1000719.

Foote, B., and J. Yoder. 2000. "Big Ball of Mud.” http://www.laputan.org.mud/ Accessed Jan 32015.

Hacking, I. 1992. "The Self-Vindication of the Laboratory Sciences." In Science as Practice and Culture, edited by A. Pickering, 29-64. Chicago, IL: University of Chicago Press.

Hacking, I. 1995. “The Looping Effects of Human Kinds.” In Causal Cognition: A Multidisciplinary Debate, edited by D. Sperber, et al., 351-394. New York: Clarendon Press.

Hartwell, L., J. Hopfield, S. Leibler, and A. Murray. 1999. "From Molecular to Modular Cell Biology." Nature 402: 47-52.

Jacob, F. 1977. "Evolution and Tinkering." Science 196: 1161-1166.

Kashtan, N., and U. Alon. 2005. "Spontaneous Evolution of Modularity and Network Motifs." Proc Natl Acad Sci USA 102:13773-13778.

Keller, E. F. 2009. "Knowledge as Making, Making as Knowing: The Many Lives of Synthetic Biology." Biological Theory 4 (4): 333-339.

Kendig, C. 2016a. Natural Kinds and Classification in Scientific Practice. New York: Routledge.

Kendig, C. 2016b. "What Is Proof of Concept Research and How Does It Generate Epistemic and Ethical Categories for Future Scientific Practice?" Science and Engineering Ethics 22(3): 735-753. doi:10.1007/s11948-015-9654-0.

Kirschner, M., and J. Gerhart. 1998. "Evolvability." Proceedings of the National Academy of Sciences USA 95: 8420-8427.

Kirschner, M., and J. Gerhart. 2005. The Plausibility of Life: Resolving Darwin's Dilemma. Yale University Press: New Haven.

Kitcher, P. 1989. "Explanatory Unification and the Causal Structure of the World." In Scientific Explanation, Vol. 10 of Minnesota Studies in the Philosophy of Science, edited by P. Kitcher and W. Salmon, 410-505. Minneapolis: University of Minnesota Press.

Knight, T. 2003. "Idempotent Vector Design for Standard Assembly of Biobricks." MIT Synthetic Biology Working Group. http://hdl.handle.net/1721.1/21168.

Longy, F. 2013. “Artifacts and Organisms: A Case for a New Etiological Theory of Functions." In Functions: Selection and Mechanisms, edited by P. Huneman, 185-211. Dordrecht: Springer.

Marlière, P. 2009. "The Farther, the Safer: A Manifesto for Securely Navigating Synthetic Species Away From the Old Living World.” Systems and Synthetic Biology 3: 77-84.

Martin, V. J., D. J. Pitera, S. T. Withers, J. D. Newman, and J. D. Keasling. 2003. "Engineering a Mevalonate Pathway in Escherichia Coli for Production of Terpenoids." Nat Biotechnol 21 (7): 796-802.

Morange, M. 2009. "Synthetic Biology: A Bridge Between Functional and Evolutionary Biology." Biological Theory 4 (4):368-377.

Nandagopal, N., and M. Elowitz. 2011. "Synthetic Biology: Integrated Gene Circuits.” Science 333: $1244-1248$.

Oftedal, G., and V.-P. Parkkinen. 2013. "Synthetic Biology and Genetic Causation." Studies in History and Philosophy of Biological and Biomedical Sciences 44: 208-216.

O’Malley, M. 2009. “Making Knowledge in Synthetic Biology: Design Meets Kludge.” Biological Theory 4 (4): 378-389. 
O’Malley, M., A. Powell, J. Davies, J. Calvert. 2008. “Knowledge-Making Distinctions in Synthetic Biology." BioEssays 30: 57-65.

Putnam, H. 1975. "The Meaning of 'Meaning.'” In Language, Mind, and Knowledge. Vol. 7 of Minnesota Studies in the Philosophy of Science, edited by K. Gunderson, 131-193. Minneapolis: University of Minnesota Press.

Rouse, J. 2003. How Scientific Practices Matter. Chicago: University of Chicago Press.

De Regt, H., S. Leonelli, and K. Eigner, editors. 2009. Scientific Understanding: Philosophical Perspectives. Pittsburgh: University of Pittsburgh Press.

Saez-Rodriguez, J., A. Kremling, and E. Gilles. 2005. "Dissecting the Puzzle of Life: Modularization of Signal Transduction Networks." Computational Chemical Engineering 29: 619-629.

Schlosser, G., and G. Wagner, editors. 2004. Modularity in Development and Evolution. Chicago: Univ. of Chicago Press.

Schyfter, P. 2012. “Technological Biology? Things and Kinds in Synthetic Biology.” Biology and Philosophy 27: 29-48.

Schaffer, J. 2005. “Contrastive Causation.” The Philosophical Review 114: 297-328.

Soler, L., editor. 2012. Characterizing the Robustness of Science: After the Practice Turn in Philosophy of Science. Volume 292 of Boston Studies in the Philosophy of Science. New York: Springer-Verlag SBM.

Soler, L., S. Zwart, M. Lynch, and V. Israel-Jost, editors. 2014. Science After the Practice Turn in the Philosophy, History, and Social Studies of Science. London: Routledge.

Sprinzak, D., and M. Elowitz. 2005. "Reconstruction of Genetic Circuits.” Nature 438 (7067): 443-8.

Houkes, W. N., and P. E. Vermaas. 2013. "Pluralism on Artefact Categories: A Philosophical Defense." Review of Philosophy and Psychology 4 (3): 543-557.

Van Fraassen, B. 1980. The Scientific Image. Oxford: Oxford University Press.

Wagner, A. 2011. The Origins of Evolutionary Innovations. Oxford: Oxford University Press.

Wagner, G., M. Pavlicev, and J. Cheverud. 2007. “The Road to Modularity.” Nature Reviews Genetics 8: 921-931.

Waters, C. K. 2007. “Causes That Make a Difference.” The Journal of Philosophy 104: 551-579.

Weber, E., R. Gruetzner, S. Werner, C. Engler, and S. Marillonnet. 2011. "Assembly of Designer TAL Effectors by Golden Gate Cloning.” PLOS One 6 (5): e19722.

West-Eberhard, M. J. 2003. Developmental Plasticity and Evolution. Oxford: Oxford University Press.

Wimsatt, W. 2009. Re-Engineering Philosophy for Limited Beings. Cambridge: Harvard University Press.

Wuensche, A. 2004. "Basins of Attraction in Network Dynamics: A Conceptual Framework for Biomolecular Networks." In Modularity in Development and Evolution, edited by G. Schlosser and G. Wagner, 307-312. Chicago: University of Chicago Press.

Wittgenstein, L. 1953. Philosophical Investigations. Edited by G. E. M. Anscombe and R. Rhees. Translated by G. E. M. Anscombe. Oxford: Blackwell.

Yu, C., Y. Cao, H. Zou, and M. Xian. 2011. "Metabolic Engineering of Escherichia Coli for Biotechnological Production of High-Value Organic Acids and Alcohols." Applied Microbiology and Biotechnology 89 (3): 573-583. 
(C) 2017 Author(s)

This is an open-access article distributed under the terms of the Creative Commons AttributionNonCommercial-NoDerivatives 4.0 International license, which permits anyone to download, copy, distribute, or display the full text without asking for permission, provided that the creator(s) are given full credit, no derivative works are created, and the work is not used for commercial purposes.

ISSN 2475-3025 